\title{
David Sherrington Commemorative Issue
}

\author{
Peter A. G. Cormack ${ }^{1}$ and David Haddleton ${ }^{2}$
}

${ }^{1}$ WestCHEM, Department of Pure and Applied Chemistry, University of Strathclyde, Thomas Graham Building, 295 Cathedral Street, Glasgow, G1 1XL, UK

${ }^{2}$ Department of Chemistry, University of Warwick, Gibbet Hill Road, Coventry, CV4 7AL, UK

This themed issue of Polymer Chemistry was commissioned following the passing, in October 2014, of Professor David Colin Sherrington, FRS. It was commissioned as a dedication to the memory of a world-class polymer chemist of international renown, but also as a celebration of a subject area which was very close to Dave's heart and upon which he founded his career and made his name. It is entirely appropriate that this collection of papers is published in a leading international journal which bears the name of the subject field with which Dave's name is synonymous and to which he contributed so much - Polymer Chemistry - but also that this collection be published in the UK by his own professional chemical society, the Royal Society of Chemistry.

Over the course of a long and illustrious academic career which began at the University of Strathclyde in Glasgow in 1971 and led to him being elected as Fellow of the Royal Society in 2007, Dave was a pioneer in a number of pure and applied polymer chemistry research areas and made unique contributions to organic and polymer chemistry. He was characterised by his scientific ingenuity, his pragmatic approach to polymer chemistry research, and his sensitivity and understanding of the technical and commercial needs of industry, but also for his unstinting support of other researchers in the community, especially young and upcoming scientists in academia and industry the World over.

The breadth of the polymer chemistry topics which appear in this themed issue reflect rather well Dave's diverse research interests, but also his willingness to tackle difficult research problems and to collaborate with others to push back the frontiers of scientific knowledge. A number of the contributors to this themed issue were fortunate enough to be under Dave's guidance as research students, whereas others have worked collaboratively with him on research programmes and benefited from his scientific knowledge and insights, his excellent hospitality and his warm sense of humour.

There is no doubt whatsoever that it is in the area of polymer-supported chemistry where his life's work has had a particularly profound impact, on both academia and industry. Dave's development of polymer-supported reagents, catalysts and sorbents helped to unite organic chemistry and polymer chemistry and led to practically useful polymeric products and processes. As but one illustration of this, Saha (10.1039/C5PY01147G) demonstrates rather well the practical advantages which accrue when metal complex catalysts are immobilised upon polymeric supports, in this particular case the immobilisation of molybdenum-based catalysts on polymer supports and the exploitation of these heterogeneous catalysts in oxidation processes using batch and continuous reactors.

The installation and exploitation of functional handles on polymer supports is an interesting topic in its own right, and there are many seminal Sherrington papers and books (e.g., Syntheses and Separations using Functional Polymers, co-edited with Phil Hodge) which teaches one how to accomplish this, however the synthesis of the supports in appropriate formats, and the control of the porous morphology, are critical parameters too, with Dave very often using suspension polymerisation-based methods to control both parameters. With respect to porous materials, microporous solids are very much in vogue and this includes polymers which are hypercrosslinked, Polymers with Intrinsic Microporosity (PIMs) and Covalent Organic Frameworks (COFs). Fontanals 
(10.1039/C5PY00771B) reviews the hypercrosslinked polymer area, and draws special attention to the synthesis and properties of these fascinating materials and their impressive performance compared to conventional materials when applied in a range of applications, e.g., as solid-phase extraction sorbents, whereas Adams (10.1039/C5PY00668F) reports upon a fundamental study in which the porosity of hypercrosslinked polymers is controlled through a systematic variation of the molar mass of the linear polystyrenes used as precursors in hypercrosslinking reactions. In the case of Adams, the linear polystyrenes were synthesised via RAFT polymerisation. Microporous polymers of a quite different type - conjugated microporous polymers (CMPs) - are reported by Vilela (10.1039/C5PY00772K). Here, the porous polymer networks are built up using Sonogashira-Hagihara cross-coupling reactions and then functionalised via post-polymerisation click chemistry. Very interestingly, the functionalised products show promise as heterogeneous catalysts in silver-catalysed heterocyclisation processes. Materials with much larger pores than those offered by microporous solids are required for 3D cell culture applications. In this regard, Cameron (10.1039/C5PY00721F) describes the synthesis of polyHIPEs using an unusual click chemistry approach. The polymers prepared in this way are described as being fully biocompatible and biodegradable, and have been shown to support cell growth in preliminary studies.

Similarly to Adams, Rannard (10.1039/C5PY00791G) uses reversible-deactivation radical polymerisation to convert vinyl monomers into interesting and useful polymeric products. ATRP is exploited as well as RAFT polymerisation, and the special role which methanol plays when used as a reaction medium for the homogeneous polymerisation of monomers such as $n$-butyl methacrylate is described. Products with controlled molar mass and molecular architecture can be prepared using the synthesis conditions disclosed. Hodge (10.1039/C5PY00272A), a long-standing collaborator and friend of Dave, uses a quite different strategy to build up polymers with interesting architecture and functionality. Three new families of macrocyclic olefins were prepared from deoxycholic acid using entropically-driven ring-opening methathesis polymerisations (ED-ROMPs). Upon functionalisation, the deoxycholic acid-containing polymers are potentially useful for supporting cell growth.

Supramolecular chemistry provides powerful tools for the preparation of complex structures and materials from relatively simple building blocks. Indeed, Dave has a series of publications in the literature where supramolecular materials chemistry has been the focus, from the self-assembly of small molecules to give liquid crystalline materials through to the exploitation of non-covalent forces to drive particle-particle assembly. Within the present collection of papers, five contributions are concerned with the harnessing of supramolecular chemistry, and self-assembly processes, to yield yet more complex structures, in both fundamental studies and work directed towards applications. Armes (10.1039/c5py00396b) exploits polymerisation-induced self-assembly for the preparation of polysulfobetaine-based diblock copolymer nanoobjects, allowing access into pure phases of spheres, worms and vesicles, and polymers with thermo-responsiveness and enhanced colloidal stability. In contrast, Besenius (10.1039/C5PY01241D) describes the preparation of phenylalanine-rich rod-like structures via the tuneable supramolecular copolymerisation of mismatched dendritic peptide comonomers; the disassembly of these smart supramolecular polymers can be triggered by $\mathrm{pH}$. Colquhoun and Hayes (10.1039/C5PY00622H) also use the supramolecular assembly of simpler building blocks to build up complexity in solution, but offer the prospect of controlling the properties of printed materials by controlling the supramolecular assembly processes using inkjet printing techniques. Somewhat reminiscent of the particle-particle assembly work reported by Sherrington, Perrier (10.1039/C5PY00912J) describes the synthesis of core-shell nanoparticles and the 2D selfassembly of the nanoparticles into well-ordered arrays; these materials have the potential to be utilised in the fabrication of low cost photonic crystals. Similarly to Perrier, Sellergren (10.1039/C5PY01318F) describes both RAFT polymerisation and self-assembly processes. However, 
in the case of Sellergren template-directed polymerisations are used to synthesise molecularly imprinted polymers. This fundamental study sheds new light on the advantages which may accrue from the use of reversible-deactivation radical polymerisation for the synthesis of molecularly imprinted polymer networks rather than the more common free radical polymerisation routes.

Facile, versatile and cost-effective are the first words which spring to mind to describe the Strathclyde Route to Branched Vinyl Polymers, a one pot, scalable methodology first disclosed by Dave in 1999. With one eye on the industrial relevance and impact of Dave's polymer chemistry legacy, Slark (10.1039/C5PY01179E) provides an industrial perspective on this polymerisation technology. In a systematic study, a series of multivinyl branchers and multivinyl chain-transfer agents are copolymerised in a variety of feed ratios to yield a number of branched vinyl polymers in high yields. The properties of the products are reported to change systematically with the composition of the monomer feed, and the results from this pragmatic modification of conventional radical polymerisation are placed in the context of reversible-deactivation radical polymerisation methodologies.

In closing, we would like to thank all of the individuals named as authors in this themed issue for their valuable contributions. Whilst the circumstances which led to the commissioning of this commemorative issue are very sad indeed, and Dave is sorely missed by his many friends and colleagues around the globe, we hope that you, the readers, enjoy tucking into this feast of worldclass polymer chemistry research. Dave would have been one of the very first to read this outstanding collection of scientific papers. 